\title{
Bandgap renormalization and excitonic binding in T-shaped quantum wires
}

\author{
M. Stopa \\ Walter Schottky Institute \\ 1 Am Coulombwall \\ D-85748 Garching, Germany \\ phone 49-89-289-12198, Fax: 49-89-289-12737 \\ e-mail: stopa@wsi.tum.de
}

(September 27, 2018)

\begin{abstract}
We calculate the electronic structure for a modulation doped and gated T-shaped quantum wire using density functional theory. We calculate the bandgap renormalization as a function of the density of conduction band electrons, induced by the donor layer and/or the gate, for the translationally invariant wire, incorporating all growth and geometric properties of the structure completely. We show that most of the bandgap renormalization arises from exchange-correlation effects, but that a small shift also results from the difference of wave function evolution between electrons and holes. We calculate the binding energy of excitons in the wire, which breaks translational invariance, using a simpler, cylindrical model of the wire. For a single hole and a one dimensional electron gas of density $n_{e}$, screening of the exciton binding energy is shown to approximately compensate for bandgap renormalization, suggesting that the recombination energy remains approximately constant with $n_{e}$, in agreement with experiment. We find that the nature of screening, as treated within our non-linear model, is significantly different from that of the various linear screening treatments, and the orthogonality of free carrier states with the bound electron states has a profound effect on the screening charge. We find that the electron and hole remain bound for all densities up to $\sim 3 \times 10^{6} \mathrm{~cm}^{-1}$ and that, as $n_{e}$ increases from zero, trion and even "quadron" formation becomes allowed.
\end{abstract}

\section{INTRODUCTION}

The gap in a semiconductor heterostructure between conduction and valence bands, and the interaction between electrons in the one and holes in the other, are known to depend in a complicated fashion on the presence of mobile charges [1] [3]. The interpretation of optical experiments in doped semiconductor quantum wires 四, for example, must invoke the variation of band edges due to many-body and geometrical effects, the "red shift" associated with electron-hole binding into excitons and finally the reduction of the exciton binding energy due to screening by free carriers.

Theoretical description of bandgap renormalization (BGR) and exciton formation and screening are frequently addressed with a many-body formalism [6, [7. Within this framework, contribution of both electronphonon and electron-electron self-energies to BGR [8] as well as the influence of dynamical screening on the exciton binding energy can be studied. The many-body treatment, however, has the disadvantage that, for BGR it commonly ignores geometrical factors, such as the quantum confined Stark effect [9], whose relevance is structure-specific. Furthermore, in the exciton problem, many-body theory treats screening within the linear approximation and, generally, influence of the bound electron on the free electrons is not fully included. In particular the orthogonality of the free electron states with the bound state, which increases in importance in lower dimensional systems, are typically not included [10].

A different formalism without these shortcomings, al- beit one which forsakes the electron-phonon interaction and the dynamics of screening, is that of density functional theory (DFT) [11]. Within DFT the ground state energy of an interacting many particle system is known rigorously to be given by a functional of the density. The essential problem of DFT is that this functional is unknown and the components beyond kinetic and direct electrostatic energy are isolated into an "exchangecorrelation" functional which must be approximated radically. Typically the limit wherein the density varies adiabatically, known as the local density approximation (LDA), is assumed. Nonetheless, successful treatments of a wide class of systems; from atoms and molecules to solids and heterostructures, abound in the literature. It is the purpose of this paper to provide a theoretical description of BGR and exciton screening, applied particularly to semiconductor quantum wires (QWs), within DFT.

We are interested in quantitative comparison with optical experiments on T-shaped quantum wires (TQWs) in the presence of a one-dimensional gas of electrons (1DEG) with 1D density $n_{e}$, induced in the conduction band via a combination of modulation doping and gating 12]. By way of warning, this one-component plasma contrasts with the case of many studies which focus on intrinsic QWs wherein an overall charge neutral electron-hole plasma is generated entirely through photoexcitation. In our case photoexcitation is assumed to provide a small number of holes (which we take as a single hole) and to have a negligible effect on the density of conduction band electrons. 


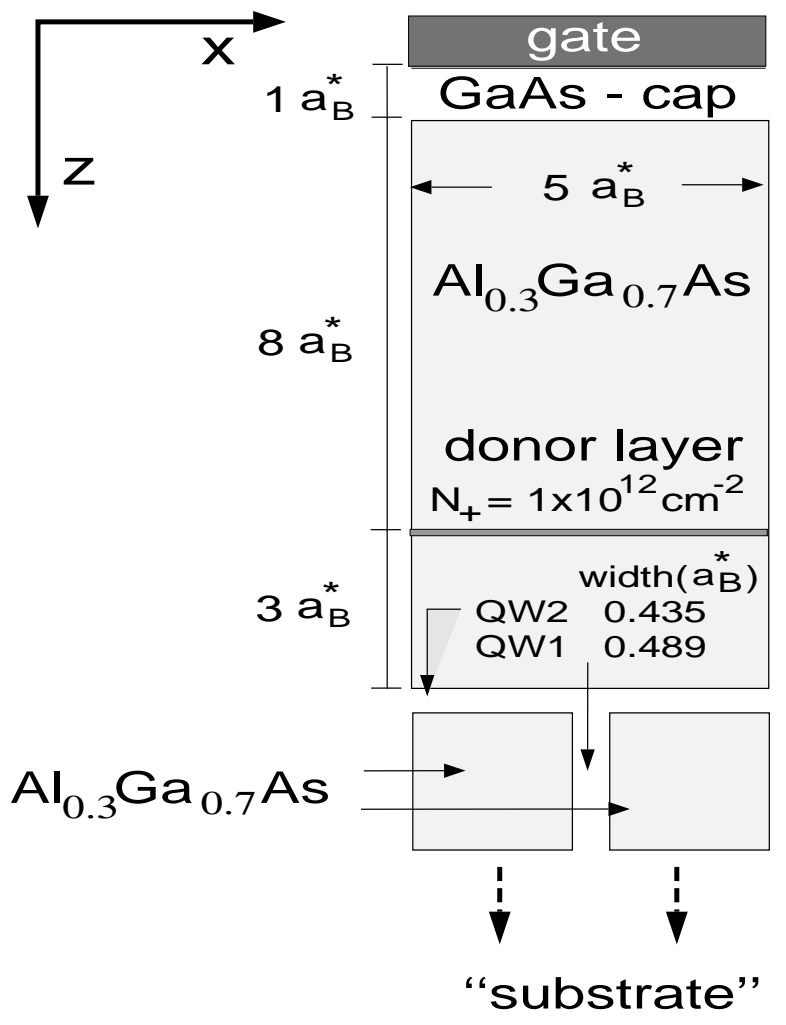

FIG. 1. Schematic of T-shaped quantum wire cross section.

We evaluate the band structure of a TQW in two steps. In the first step (section II) we consider a realistic model of a cross-section of a TQW, shown in Fig. 1, and solve self-consistently for the electronic structure of the subbands, as a function of the gate voltage, assuming translational invariance along the wire. Material-specific effective masses and band offsets, as well as the actual growth profiles and doping densities of the structure are included. The exciton problem, which breaks translation invariance, is treated in the second step (section III) by a simplified, cylindrical model of a quantum wire which employs the results of the first step for its rationale and its parameters. Thus the philosophy of the calculation is that the exciton formation and its screening represent an additive correction to the translationally invariant band structure of step 1. This exciton effect can be analyzed within a single subband, generic wire model so long as the effects of lateral confinement are correctly borrowed from the more faithful, albeit 2D calculation in step 1 .

Among our main results are the following. For the translationally invariant band structure (section II): (1) We find a BGR as a function of $n_{e}$ with comparable order of magnitude to that derived within many-body theory. (2) One small $(\sim 20 \%)$ component of this BGR arises from a variation of the lowest electron subband eigenfunction with $n_{e}$. Specifically, at higher $n_{e}$ the lowest electronic wave function spreads somewhat along the overgrowth interface (throughout QW2 in Fig. 1) whereas it is more strongly confined at the junction (i.e. the intersection of the two wells) at lower $n_{e}$. The hole wave function, by contrast, is always localized at the junction. (3) The stability of the hole eigenstate at the junction is dependent on the boundary conditions used for large $z$ (see Fig. 1). If the chemical potential is pinned to an assumed background donor density in the substrate (we use the term "substrate" to denote the region far from the donor plane, i.e. large $z$, see Fig. 1), then the hole bound state becomes a resonance which is metastable to escape into the substrate. On the other hand, when the substrate is treated as exactly electrically neutral (or pinned to acceptors) the hole state at the junction is stable. (4) For the specific dimensions and compositions which we consider [12] the wires are predominantly one-dimensional with filling of no more than two subbands and spreading of the density into QW2 generally less than $\sim 15 \%$ of the total density.

Regarding the exciton problem (section III), we find: (1) the exciton binding energy weakens considerably as the 1DEG density is turned on, but never vanishes completely. (2) The requirement of orthogonality between the bound electron eigenfunction and those of the screening electrons leads to an anti-screening of the exciton, such that the density of free electrons in the vicinity of the hole is depressed. This effectively positive charge raises the energy of the hole while the energy of the electron actually becomes more negative and its localization about the hole increases with density.

Finally, the combination of the bandgap renormalization and the exciton screening tend to cancel and, consistent with experiments, the recombination energy remains relatively constant with $n_{e}$. This result is plotted in figure 6 but discussed at the end of section III.

We principally employ effective atomic units wherein $1 R y^{*}=m^{*} e^{4} / 2 \hbar^{2} \kappa^{2} \approx 5.25 \mathrm{meV}$ and $1 a_{B}^{*}=$ $\hbar^{2} \kappa / m^{*} e^{2} \approx 100 \AA$. For comparison with experiments we also use $\mathrm{cm}^{-1}$, where $1 \mathrm{a}_{B}^{*-1} \approx 10^{6} \mathrm{~cm}^{-1}$.

\section{TRANSLATIONALLY INVARIANT WIRE: BGR}

We calculate the electronic structure of the GaAs AlGaAs TQWs [13] by solving Schrödinger and Poisson equations self-consistently, within a region illustrated in Fig. 1, for the conduction band electrons, and by including exchange and correlation in the local density approximation (LDA) 11]. There are numerous calculations of BGR in lower dimensional systems which employ many-body theory to determine the self-energy correction to the subbands from exchange and correlation effects [14,15]. In DFT a bandgap renormalization arises as follows. First, variations in the electrostatic potential tend to affect both bands equally except for kinematic effects like the quantum confined Stark effect [9] where the 
electron wave function is centered on a different spatial location (at different potential) than that of the hole. By contrast, exchange and correlation effects lower the energy of electrons in proportion to $n_{e}$, the electron density, and raise the energy of holes in proportion to $n_{h}$, the hole density, producing an overall shrinkage of the bandgap. For the one component plasma considered here, therefore, the exchange-correlation potential acts only on the conduction band electrons, since $n_{h} \approx 0$.

One advantage of our procedure is that while manybody calculations in principle begin from wave functions and subband eigenenergies derived from some Hartree calculation, the variation of these properties with density and the aforementioned kinematical corrections to the bandgap are usually ignored. By contrast DFT automatically includes these. Furthermore, the separate contributions from kinematic effects as opposed to exchange and correlation are easily isolated (to first order) by performing a calculation without the exchange-correlation potential (i.e. Hartree only).

We employ the parameterization of Ceperley and Alder [16] for the density-dependent, exchange-correlation potential $V_{x c}(n(\mathbf{x}))$. Taking $y$ as the translationally invariant direction (see figure 1 ), the Schrödinger equation in the $x-z$ plane $\left(k_{y}=0\right)$ reads

$$
\begin{aligned}
{\left[-\nabla \frac{1}{2 m^{*}(x, z)} \nabla+V_{C}(x, z)+e \phi(x, z)+\right.} \\
\left.V_{x c}(n(x, z))\right] \psi_{n}(x, z)=E_{e}^{n} \psi_{n}(x, z)
\end{aligned}
$$

where $V_{C}(x, z)$ is the conduction band offset 17] which depends only on the local aluminum concentration $\eta$ (we use the symbol $\eta$ instead of the standard $x$ to avoid confusion with the coordinate $x$ ), the $2 \mathrm{D}$ eigenfunctions and eigenvalues are given by $\psi_{n}(x, z)$ and $E_{n}$ respectively, and the electrostatic potential is $\phi(x, z)$. Also in equation 11 the level index is $n$, and the subscript $e$ refers to electrons in the conduction band whereas for holes we use $E_{h}^{n}$. The eigenstates are abbreviated below as $e n$ and $h n$ for electrons and holes, respectively. Periodic boundary conditions are taken at $x= \pm a / 2$ where $a=5 a_{B}^{*}$ is the period of the TQW superlattice as fabricated [12]. The effective mass dependence on position arises through its dependence on $\eta$ [17]. In the specific device we consider, all regions have either $\eta=0$ (pure GaAs) or else $\eta=0.3$ for the barrier regions. We ignore interface grading effects as well as image effects, taking the dielectric constant as $\kappa=12.5$ everywhere.

For the solution of Poisson's Equ. we also assume periodic boundary conditions at the $x$-borders. For the surface $z=0$ we simulate the surface metal gate in the standard fashion [13] by Dirichlet boundary conditions which fix the gate potential modulo an offset of $0.8 \mathrm{eV}$ for the Schottky barrier. Variation of the electron sheet density in the overgrowth well $N_{e}$ (or, equivalently, the $1 \mathrm{D}$ density $n_{e}$ ) is accomplished by biasing this surface gate. Other experimental methods for varying $n_{e}$ include illumination or a variation of the thickness of the spacer between the modulation doping layer and the overgrowth well. While we have modeled each of these methods independently, in fact in each case there is an approximately homogeneous sheet (or sheets) of positive charge balancing the electronic charge in the wire/well and the difference in the electronic structure between the various methods, for a given resultant $n_{e}$, is negligible. Thus here we actually vary a surface gate voltage to change density, but in displaying the results it is sufficient just to plot variables versus $n_{e}$.

For large $z$ we find, as noted, that the solution for the holes is sensitive to the asymptotic value of the potential. Generally, we assume Neumann boundary conditions, which is equivalent to assuming complete neutrality of the substrate. However we discuss below the nature of the hole metastable state in the case where the chemical potential is pinned to a shallow donor level at large $z$.

Since the modulation doping is $n$-type, the Fermi level is close to the conduction band throughout the device. Furthermore, since even during photoluminescence measurements, the excitation power is very low, we ignore the valence band contribution to the charge density. Therefore, the total density which enters the Poisson equation is given as

$$
\rho(x, z)=N_{+}(x, z)+n(x, z)
$$

where $n(x, z)$ is the density of conduction band electrons only, and where the charge density of ionized donors is $N_{+}(x, z)$. The conduction band electron density is given in the well regions by

$$
n(x, z)=\int d k \sum_{n} f\left(\mu-k_{y}^{2}-E_{n}\right)\left|\psi_{n}(x, z)\right|^{2}
$$

where $f$ is the Fermi function, $\mu$ is the chemical potential (assumed constant throughout the device and taken as the zero of energy) and $k_{y}^{2}$ is the kinetic energy of motion in the $y$-direction. We also employ a ThomasFermi approximation for the density of electrons in the barrier regions away from the wells. Finally, we assume temperature $T=4 \mathrm{~K}$.

We find little difference in the results whether the donors, which are silicon atom DX centers [18], are placed in thermal equilibrium with the electron gas or are treated as a simple sheet of positive charge with fixed areal density. Insofar as at low temperatures the latter approximation is more physical, we use this assumption in the calculations discussed in this paper.

Upon obtaining a self-consistent solution of Schrödinger and Poisson equations for conduction band electrons, we calculate the hole eigenfunctions with the potential

$$
V_{h}(x, z)=V_{V}(x, z)-e \phi(x, z)
$$


where now $\phi(x, z)$ is the electrostatic potential which solves the self-consistent problem above. The valence band offset $V_{V}(x, z)$ also depends on the aluminum concentration. We assume a bandgap offset parameter $Q_{e}=$ 0.6. We treat the holes in the simplest approximation, assuming a single, heavy-hole isotropic effective mass which depends only on aluminum concentration; specifically $m_{h}^{*}(G a A s)=0.377 m_{0}$ and $m_{h}^{*}\left(A l_{0.3} G a_{0.7} A s\right)=$ $0.403 m_{0}$ [19].

To summarize therefore and to make the context of this calculation clear, we are interested principally in the BGR as a function of $n_{e}$, the integral over $x$ and $z$ of $n(x, z)$, which we imagine to be modulated experimentally with an electrostatic gate on the overgrowth surface [12]. Thus we solve the evolving self-consistent electronic structure assuming the chemical potential to be far from the valence band (i.e. no holes). We solve for the hole subbands as a one particle problem after solving the electronic structure at given $n_{e}$, using the resultant electrostatic potential. We use a simplified band structure for the holes, in contrast to ref. [20] which employs the full, four-band Luttinger Hamiltonian but which assumes $n_{e}=0$.

\section{A. Electronic structure}

The parameters for the calculation are summarized in the schematic, Fig. 1. We follow reference 20] by designating the overgrowth quantum well as "QW2" and the initial growth well as "QW1."

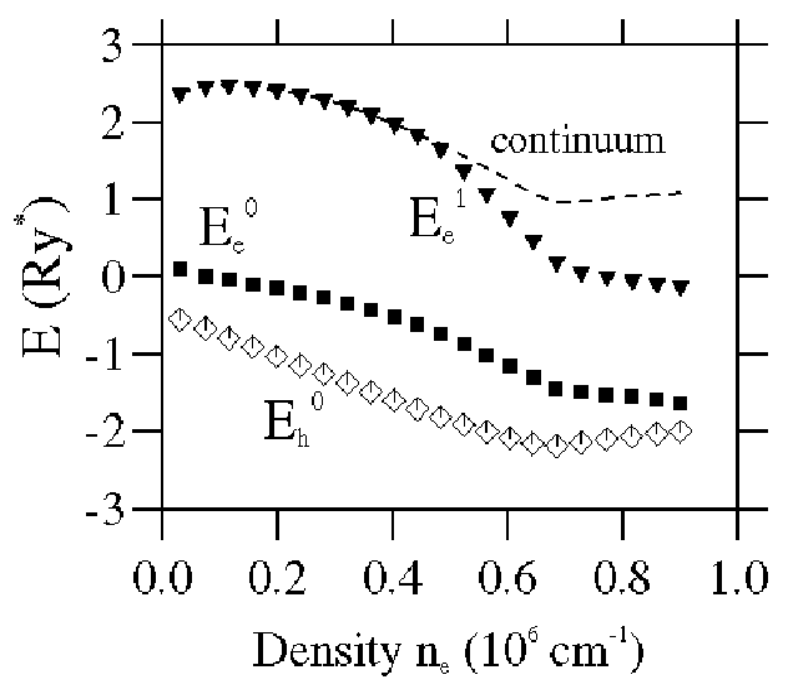

FIG. 2. Subband energies vs. wire 1D density for electron (e) and hole (h). Electron levels measured with respect to Fermi surface; hole level with respect to escape to substrate. Electron continuum begins at dashed line. Filling of $E_{e}^{1}$ begins at $n_{e} \sim 0.5$.

The structure of the subbands of conduction band elec- trons as a function of $n_{e}$ is shown in Fig. 2. The zero of energy for the electrons is the Fermi energy $E_{F}$ (the plotted hole energy is discussed further below). In the higher density regime a second subband, which has concentration in the inter-wire QW2 region (cf. Fig. 4), is occupied. The region marked "continuum" corresponds to the beginning of a dense set of states which are asymptotically free to escape into the substrate (i.e. along QW1 which runs in $+z$ direction). This is so because QW1 is wider than the QW2, and the electrostatic advantage of proximity to the positive dopants in QW2 is overcome by the additional confinement energy there. Nonetheless, the spreading of the occupied states, when the total density is increased, occurs within QW2 (so as to compensate the positive charge of the donors and gate).

It is noteworthy that for $n_{e} \gtrsim 0.5 a_{B}^{*-1}(\approx 0.5 \times$ $\left.10^{6} \mathrm{~cm}^{-1}\right)$, there are two subbands which are below the continuum in contrast to the situation for lower $n_{e}$ where only a single bound state exists. This is also consistent with the bare $\left(n_{e}=0\right)$ TQW studied in Ref. 20 which had a nearly identical aspect ratio (i.e. ratio of well widths) and exhibited only a single state separate from the continuum. The estimate there of a separation between $E_{e}^{0}$ and continuum of $\sim 15 \mathrm{meV}$ is also consistent with our slightly lower value of $\sim 13 \mathrm{meV}$. Interestingly, this separation remains approximately constant (Fig. 2) as $n_{e}$ is increased, until $E_{e}^{1}$ drops down from the continuum and begins to fill.

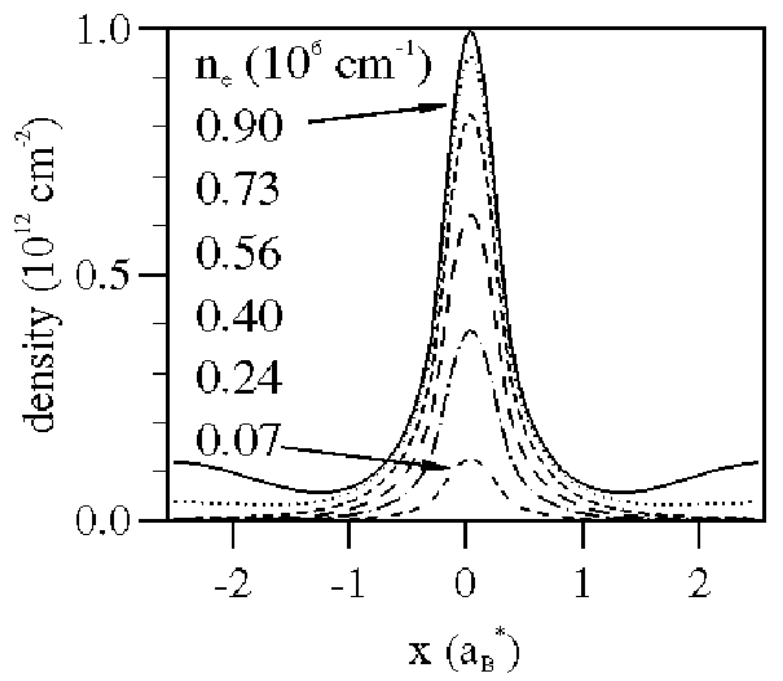

FIG. 3. Areal density (integrated along $z$ ) vs. $x$ for various total wire densities. Confinement to wire almost complete below $n_{e} \sim 0.6$. For highest $n_{e}$ interwire density develops subsidiary maximum at $\pm 2.5 a_{B}^{*}$.

In Fig. 3 we have plotted the $2 \mathrm{~d}$ surface density as a function of $x$ for various total $n_{e}$. As $n_{e}$ approaches $1 a_{B}^{*-1}$ some spreading of the density away from the Tjunction occurs. This results both from the occupation of $e 1$ and also from slight wave function spreading of $e 0$ 
(cf. Fig. 4). Note, however, that, rather than spreading smoothly out from the wire, the density achieves a local maximum at an inter-wire minimum in the potential (at $\pm 2.5 a_{B}^{*}$ which, due to periodicity, are equivalent points), analogous to the electrostatic potential minima in the barriers of a semiconductor superlattice. Here, however, the effect is both electrostatic and quantum mechanical. Note, in figure 4, where the moduli squared of the eigenfunctions are plotted, that the subband $e 1$ has two nodes along the $x$-direction (i.e. along QW2). For different parameters (not shown), such as for a wider QW2 or much higher $n_{e}$, a state with a single node at the T-junction is lower in energy. But in this regime the attractiveness of the T-junction is sufficient to stabilize the even node state.
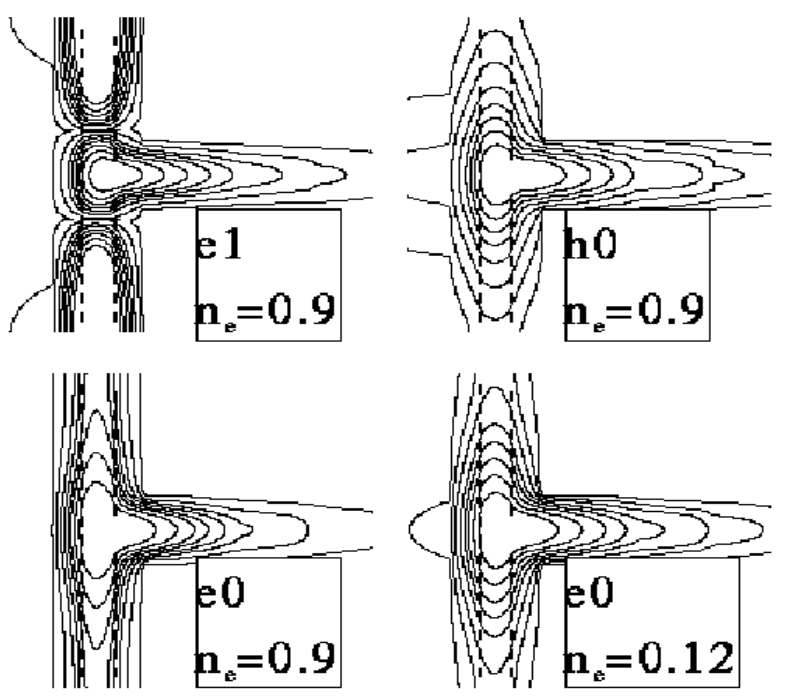

FIG. 4. Wave functions (moduli squared) showing spreading of $\mathrm{e} 0$ as $n_{e}$ increases from $0.12 \times 10^{6} \mathrm{~cm}^{-1}$ (LR) to $0.9 \times 10^{6} \mathrm{~cm}^{-1}(\mathrm{LL})$. First excited electron state at shows two nodes along $x$ (UL). Hole ground state (UR) unchanging and strongly confined at junction.

The spreading of $e 0$ with increasing $n_{e}$ observable in Fig. 4 is relevant to the evolution of the gap between conduction and valence bands. We will see below that the dominant portion of the BGR arises from exchange and correlation effects. Nonetheless, as noted in the introduction, the difference between the evolution of electron and hole wavefunctions with $n_{e}$ produces a kind of kinematic BGR as in the quantum confined Stark effect. Explicitly, the hole eigenfunction, which is shown in Fig. 4 only for $n_{e}=0.9 \times 10^{6} \mathrm{~cm}^{-1}$, undergoes essentially no change with $n_{e}$ over the $n_{e}$ range of Fig. 2. Therefore the hole essentially tracks the electrostatic potential at the T-junction. The electrons in $e 0$ however, for increasing $n_{e}$, are able to lower their energy by spreading along QW2. This implies a redshift in the bandgap which emerges even in the absence of exchange-correlation effects (see the Hartree results in Fig. 6 below).

\section{B. Hole states}

The stability of the hole state at the T-junction depends weakly on the chosen boundary conditions for Poisson Eq. at large $z$. If we pin the chemical potential to an assumed shallow donor level at large $z$, a common assumption, the hole state at the T-junction is merely metastable, and escape, through a long, shallow barrier, to the substrate is energetically preferred. Assuming Neumann boundary conditions, or "flat bands" at large $z$, on the other hand, results in a true bound state. Dynamically, the difference between "pinned band" and flat band conditions is mostly inconsequential for holes photo-generated at the junction. For any reasonable background (i.e. un-intentional) donor density, the barrier to escape of the hole is too large and the hole will remain and, presumably, recombine in the wire. Furthermore, the hole will induce an image in the electron gas which will cause further, electrostatic binding to the wire region. A similar effect of holes bound to a 2DEG has been discussed recently in Ref. [21].

For holes generated in the substrate, far from the wire, however, the dynamics of the diffusion of holes into the wire region can be realistically expected to depend on the band shape as reflected in this dichotomy over boundary conditions. Generally, fewer background donors is favorable to hole diffusion from substrate into wire.

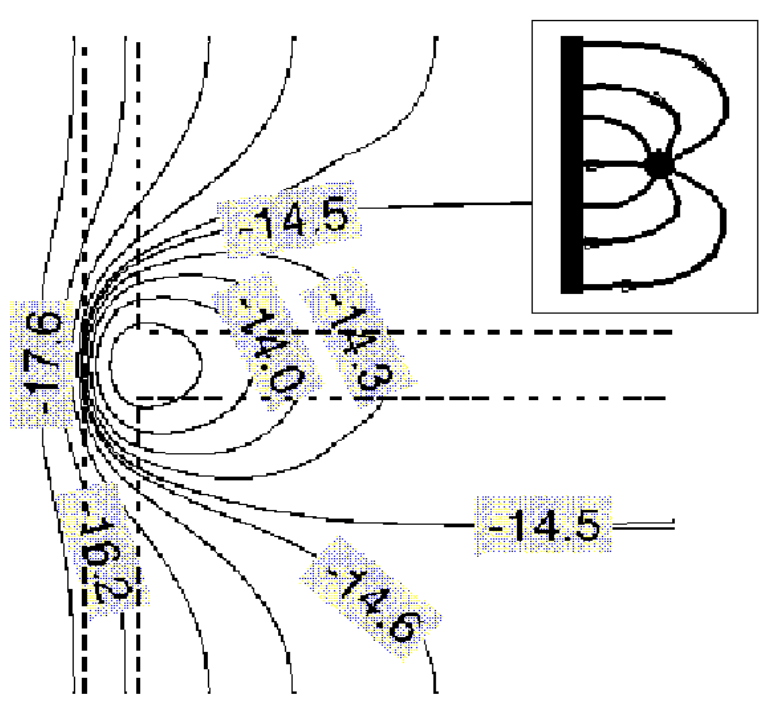

FIG. 5. Contour plot of electrostatic potential for $n_{e}=0.9 \times 10^{6} \mathrm{~cm}^{-1}$. Schematic illustrates field lines, which help to localize hole at junction.

Even for pinned band conditions the basic charge distribution near the wire establishes a purely electrostatic barrier to hole escape, as seen in Fig. 5. Here we plot the $2 \mathrm{~d}$ electrostatic potential for $n_{e}=0.9 \times 10^{6} \mathrm{~cm}^{-1}$. The potential contour principally derives from a plane of 
positive charge (the donor layer) and the line of negative charge in the wire. This results in an electrostatic potential hill near the junction (to which holes are attracted). Thus the hole has at least a long shallow barrier to escape even before considering the quantum effect that further binds the hole to the junction.

In Fig. 2 we have plotted the energy of the first hole subband, $E_{h}^{0}$, versus $n_{e}$. In this case we have used Neumann boundary conditions for Poisson's Eq. at large $z$ and the energy zero for $E_{h}^{0}$ is the electrostatic potential at large $z$ added to the confinement energy of QW1; in other words it is the energy required to escape to the substrate continuum. Here we see that the state is stable, and not merely metastable. Further, the increase in the binding with $n_{e}$ (up to $n_{e} \sim 0.6 \times 10^{6} \mathrm{~cm}^{-1}$ ) arises from the strengthening of the electrostatic potential minimum (for holes) shown in Fig. 5.

\section{Bandgap}

The bandgap in the heterostructure depends upon the assumed intrinsic bandgap in the host materials, GaAs and $A l G a A s$ which, as noted, we have taken from the literature [17]. The bandgap variation with $n_{e}$ is obtained from a sum of the lowest subband energies for the electron and the hole, each measured with respect to the GaAs band edge at any common point, then added to the intrinsic bandgap. Figure 6 illustrates the variation of the bandgap as a function of $n_{e}$. The rapid change for small $n_{e}$ for the full LDA calculation derives from the form of the exchange-correlation potential. The exchange energy alone, for comparison, is proportional to the Fermi momentum $k_{F}=\sqrt{E_{F}}$ [22]. The overall order of magnitude and shape of the BGR are consistent with the results of calculations within the GW approximation [8]. However, since the authors of reference [8] assume a neutral, two-component plasma, the results are not quantitatively comparable.

Also in figure 6 we plot the BGR for the case where the exchange-correlation potential is turned off, that is, for pure Hartree. Here the bandgap still varies, albeit much less, with $n_{e}$. The origin of the shift, alluded to in the discussion of Fig. 4, is the variation of the wavefunction of the electron with $n_{e}$ which causes a kinematic component to its energy while the hole eigenstate is essentially dormant at the junction. This effect is a slight generalization of the quantum confined Stark effect, wherein the bandgap is affected by an electric field, which is varied, between the electron location and that of the hole. In our case the electron additionally shifts its location as the gate bias varies.

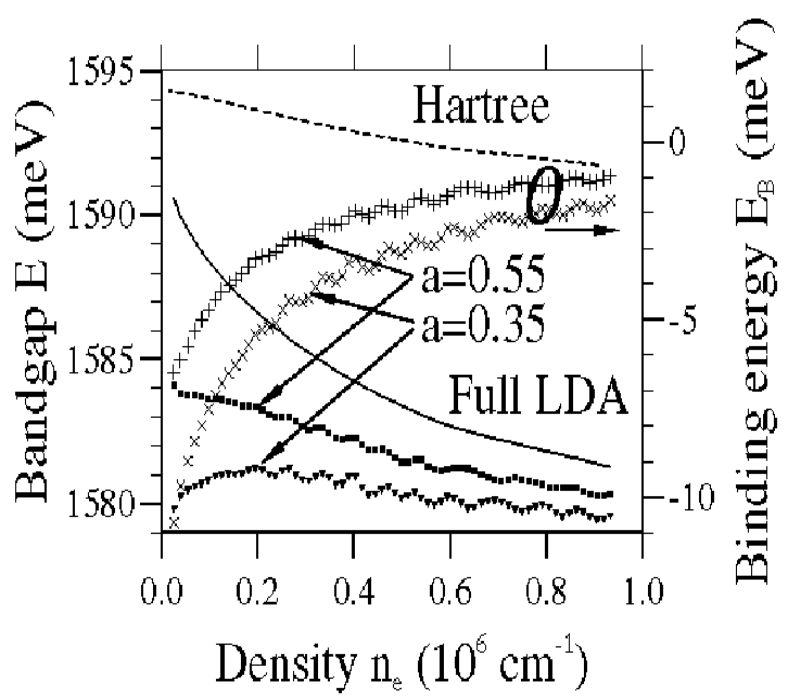

FIG. 6. Bandgap renormalization for full LDA (solid) and for pure Hartree (dashed), here in $\mathrm{meV}$, as a function of density. Exciton binding energies for wire radius $a=0.55 a_{B}^{*}$ (pluses) and $a=0.35 a_{B}^{*}$ (crosses) and LDA bandgap corrected for excitonic energy as squares and triangles, respectively.

We postpone a discussion of the final result of Fig. 6, the behavior of the BGR when correction for excitonic energy and screening are included, until after the next section.

\section{EXCITON}

The electronic structure of the exciton can be treated in a fashion analogous to the calculation of electron binding to shallow impurities, which was described some time ago by Vinter [23] for a silicon inversion layer. In contrast to the impurity problem though, the hole cannot be treated as a point charge, but has a wave function which must be computed self-consistently with those of the bound and free electrons.

In this section we employ a simplified geometry, shown in Fig. 7, for a single mode, cylindrical quantum wire along direction $y$ of radius $a$ surrounded by a cylindrical metal gate at radius $R$ whose potential $U_{\text {ext }}$ is used to vary the electron density. The radial charge distribution of both electrons and holes is assumed to be given by a single Gaussian wave function $\xi_{1}(r)=\frac{1}{a} \sqrt{\frac{2}{\pi}} e^{-r^{2} / a^{2}}$. The eigenstates of the electrons are determined by

$$
\left(-\frac{1}{2 m_{e}} \frac{\partial^{2}}{\partial y^{2}}+V_{e f f}(y)+\tilde{V}_{x c}(y)\right) \psi_{p}(y)=\varepsilon_{e}^{p} \psi_{p}(y),
$$

where

$$
V_{e f f}(y) \equiv \int d^{2} \mathbf{r}\left|\xi_{1}(r)\right|^{2} e \phi_{e}(r, y)
$$


and where the electron effective mass is $m_{e}$ and $\phi_{e}(r, y)$ is the electrostatic potential (see below). The length of the cylinder is $L_{y}$ and Eq. 5 is treated with periodic boundary conditions at the ends $y= \pm L_{y} / 2$. The effective $1 \mathrm{D}$ exchange correlation potential at $y$ is determined from the $3 \mathrm{D}$ parameterized form by

$$
\tilde{V}_{x c}(y)=2 \pi \int d r r \mathbf{r} \rho_{e}(r, y) V_{x c}\left(\rho_{e}(r, y)\right)
$$

where $\rho_{e}(r, y)=\left|\xi_{1}(r)\right|^{2} \tilde{\rho}_{e}(y)$ and where $\tilde{\rho}_{e}(y)$ is determined by filling eigenstates of Eq. 5 up to a fixed chemical potential $\mu$, taken as the energy zero of the problem.

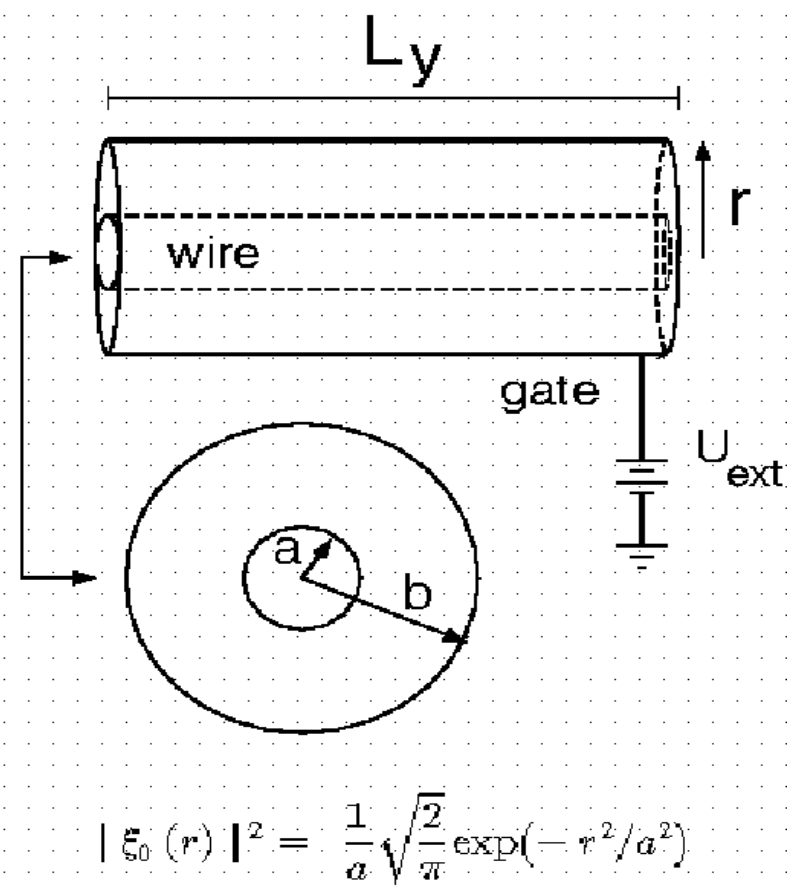

FIG. 7. Schematic of cylindrical wire for model exciton calculation. Hole centered at midpoint of wire. Radial charge distribution of hole and electron taken as single Gaussian. Drawing not to scale: $b \stackrel{\sim}{\sim} 10 a$.

The electrostatic potential in 6 is determined by solving Poisson's equation

$$
-\nabla^{2} \phi_{e}(r, y)=\frac{4 \pi}{\kappa} \rho(r, y)
$$

subject to Dirichlet boundary conditions at the gate, $r=R$, and Neumann conditions at $y= \pm L_{y} / 2$. Here $\rho(r, y)$ is the charge density of both electrons and the single hole.

An expression similar to Eq. 5, without the exchangecorrelation potential, and with the hole mass $m_{h}$ in place of $m_{e}$, can be written down for the hole, where only the lowest eigenstate is filled. Note however that in order to avoid self-interaction of the hole, the electrostatic potential (and the $y$-dependent effective potential) in this case is calculated from $\rho_{e}(r, y)$, i.e. the density of only the conduction band electrons, and is denoted $\phi_{h}$. Selfinteraction of the electrons is assumed to be compensated for by the exchange-correlation potential, as is standard in DFT; although it is known that the cancellation in $L D A$, unlike Hartree-Fock, is not exact. Note that a small inaccuracy of the model is that, by using, for the hole, the electrostatic potential computed without the hole charge $\rho_{h}(\mathbf{r})$ included, the image charge induced by the hole on the gate, with which the hole physically does interact, is absent. A more precise, though computationally taxing, approach would be to compute $\phi_{h}(r, y)$ from all the charge but then subtract $\int d^{3} \mathbf{r}^{\prime} \rho_{h}\left(\mathbf{r}^{\prime}\right) \frac{e}{\left|\mathbf{r}-\mathbf{r}^{\prime}\right|}$, the potential of the hole in free space.

The binding energy $E_{B}$ of the exciton is defined as the difference in the total energy between the case where the hole is localized, on the one hand, and the case where the single hole charge is spread uniformly in $y$, a free hole, on the other hand. The total energy must include, in addition to a sum of occupied eigenvalues, a doublecounting correction as well as the energy related to the gate. Furthermore, this gate energy is modified by the work supplied to the gate [13.24, and is hence really a free energy. The overall expression for this free energy is thus

$$
\begin{aligned}
F\left(U_{e x t}\right) & =\varepsilon_{h}^{1}+\sum_{p} n_{p} \varepsilon_{e}^{p} \\
& -\frac{1}{2} \int d^{3} \mathbf{r}\left(\rho_{e}(\mathbf{r}) \phi_{e}(\mathbf{r})+\rho_{h}(\mathbf{r}) \phi_{h}(\mathbf{r})\right) \\
& +\int d^{3} \mathbf{r} \rho_{e}(\mathbf{r})\left(\varepsilon_{x c}\left(\rho_{e}(\mathbf{r})\right)-V_{x c}\left(\rho_{e}(\mathbf{r})\right)\right) \\
& -\frac{1}{2} Q U_{e x t}
\end{aligned}
$$

where $Q$ is the total charge induced on the gate, determined by computing the normal derivative of the potential at the gate surface, and where $n_{p}$ is the occupancy of state $p$ given by a Fermi function. Also, the third term on the right hand side of Eq. 9 is a form of double counting correction for the exchange-correlation energy, where $\varepsilon_{x c}(\rho)$ is the exchange-correlation energy per particle of a homogeneous electron gas of density $\rho$ [1].

We note that one might write the total energy as simply the sum of occupied Kohn-Sham energies (including the hole) via Koopman's theorem. To lowest order, where only a single bound electron and the hole energies are changing appreciably, one could further estimate this as simply $F\left(U_{e x t}\right)=\varepsilon_{h}^{1}+\varepsilon_{e}^{1}$. However these are both approximations and the correct formula, Eq. 9, must be used for all but heuristic purposes.

\section{A. Results}

In figure 8 we show $E_{B}$ computed as a function of $U_{\text {ext }}$ for $a=0.35,0.45$ and $0.55 a_{B}^{*}$. Also plotted is the 


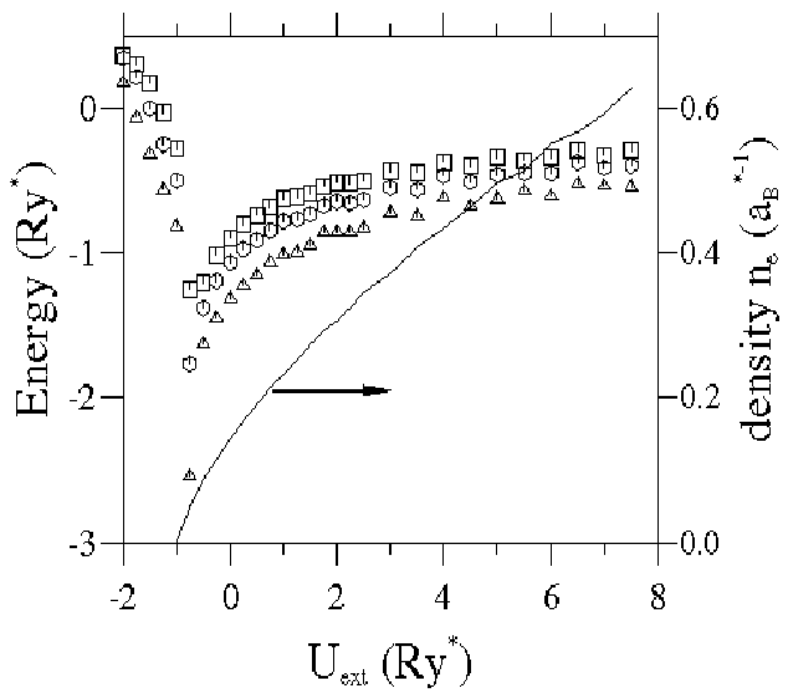

FIG. 8. Binding energy of exciton vs. gate potential for $a=0.35$ (triangles) 0.45 (hexagons) and $0.55 a_{B}^{*}$ (boxes). Line gives corresponding $n_{e}$ (see text for exact definition). Rise of energy below $n_{e}=0$ caused by shift of electronic charge to gate for delocalized hole case.

conduction band density $n_{e}$, which is here defined as the total number of conduction band electrons in the wire for the localized hole case, minus unity (the bound electron) divided by the wire length $L_{y}$. When the gate voltage becomes negative, all electrons become depleted from the

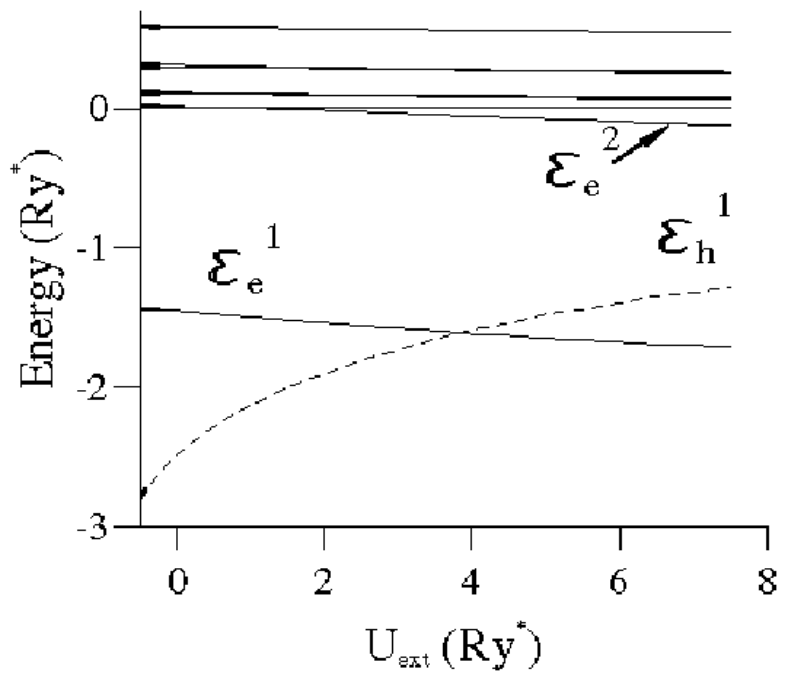

FIG. 9. Discrete Kohn-Sham energy levels for electron (solid) and hole (dashed) vs. gate voltage, $a=0.35 a_{B}^{*}$. To lowest order exciton binding energy is $E_{B} \approx \varepsilon_{e}^{1}+\varepsilon_{h}^{1}$, hence binding weakens with increased $U_{e x t}\left(\right.$ and $\left.n_{e}\right)$. Hole localizes two electrons above $U_{\text {ext }} \approx 1 R y^{*}$.

wire and, below $U_{e x t}=-1.0 R y^{*}$, the conduction electrons for the delocalized hole case shift from the wire to the gate. This explains the jump in $E_{B}$ below depletion. The minimum of $E_{B}$ occurs at the lowest density before this shift occurs, corresponding to a single electron in the wire. Clearly the minimum depends somewhat sensitively on the choice of $a$, and the binding can become quite strong for very narrow wires, as is well known [25]. The small fluctuations visible in the energies result from the finite size $L_{y}$ and the discreteness of the "free" states.

Of considerable interest is the absence of a binding to unbinding transition for the exciton. Even when we extend the density range to $>2 a_{B}^{*-1}$ (i.e. $>2 \times 10^{6} \mathrm{~cm}^{-1}$ ) and even for the widest charge distribution, $a=0.55 a_{B}^{*}$, the ground state remains bound (not shown). In fact, as shown in Fig. 9 (see also Fig. 10), a second electronic state becomes localized about the hole above $U_{\text {ext }} \approx$ $1.25 R y^{*}$, corresponding to $n_{e} \approx 0.2 a_{B}^{*-1}$, thus forming a so-called trion [26]. Here we have plotted the (KohnSham) level energies for the localized hole case, measured relative to the band edge far from the hole, so that an energy above zero indicates an asymptotically free particle. We find that for even higher densities, $>8 \times 10^{6} \mathrm{~cm}^{-1}$, a third electron can become bound to the hole; a state which we refer to naturally as a "quadron."

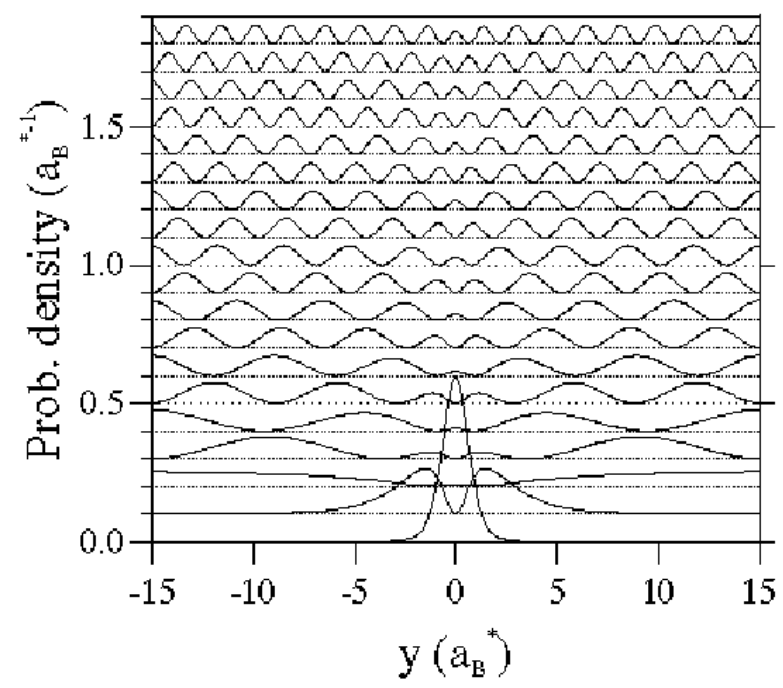

FIG. 10. Occupied electronic wave functions (moduli squared), states 1 through $19, a=0.35 a_{B}^{*}, n_{e}=0.6 a_{B}^{*-1}$. Two states are localized near hole (at $y=0)$. Orthogonality of remaining states to bound states suppresses screening charge density near hole.

The most intriguing feature of the results in Fig. 9 is that, while the hole becomes more weakly bound, and consequently more spatially extended, the electrons, particularly the lowest state, become more strongly bound with increasing $U_{e x t}$ (and consequently increasing $n_{e}$ ). A similar result for electron binding to an ionized impurity in a 2DEG silicon inversion layer was found by Vinter [23]. This is rather counter-intuitive, since one expects screening, by free electrons, of the interaction between hole and bound electron to weaken the attraction and separate the particles. Partially this result is understood 
as relating to the direct product nature of the exciton state when expressed in DFT, as opposed to the single composite state of the two-body or screened two-body problem. Thus the binding energy is not a single eigenvalue, or even the sum of two eigenvalues, but rather it must be understood as a difference between two interacting ground states. Nonetheless the decreased energy and increased localization of the lowest Kohn-Sham level seems puzzling. In figure 10 we show, for a relatively high density, $n_{e}=0.6 a_{B}^{*-1}$, the set of all occupied eigenfunctions (moduli squared). At this $n_{e}$ the lowest two electron states are localized near the hole at $y=0$. Note, however, that due to the restraint of orthogonality, the densities of all other states in the vicinity of the hole are suppressed. Thus while the total conduction band

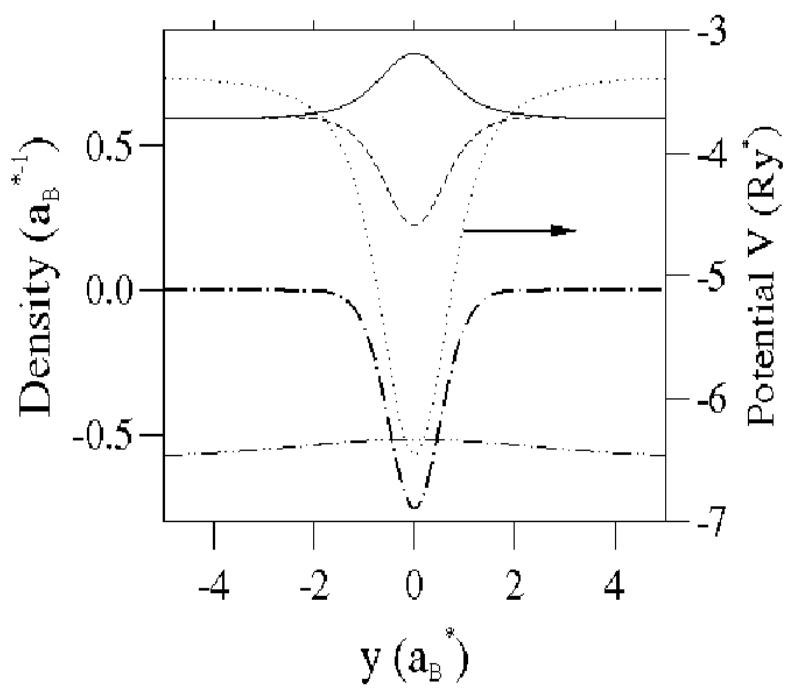

FIG. 11. Total electron density (solid), total density minus bound state (dashed), effective electrostatic potential $V_{e f f}(y)$ (dotted), density of hole (dot-dash) and density of gate charge (double-dot-dash) versus $y$, same parameters as Fig. 10.

density, exhibited in Fig. 11, peaks at $y=0$, this density minus that of the lowest state diminishes at the hole. Combined with the uniformly increasing background positive charge on the gate, this comprises a build-up of positive charge near the hole as $U_{e x t}$ and $n_{e}$ are increased. Therefore an increased density of electrons produces an effectively anti-screening effect which initially drives the electron closer to the hole and then admits a second and even a third bound electron. The hole eigenstate, meanwhile, is growing increasingly de-localized and the faster rise of its energy accounts for the increase of the overall energy (i.e. a decrease of the negative binding energy).

\section{B. Combined results}

Finally, in combining the results from Section II and Section III, we simply add the excitonic binding energy to the bandgap calculated in LDA for the translationally invariant wire. The results for this are shown in Fig. 6. Clearly the exchange-correlation and kinematic effects causing the BGR produce a red shift which is partially negated by the shift of the exciton binding energy due to screening. This is consistent with the notable insensitivity of the photoluminescence line position in optical experiments on quantum wires to either photoexcited electron-hole plasma [4,5] or to gate-generated 1DEG [12]. Interestingly, it is also consistent with recent calculations of the dynamically screened Bethe-Salpeter equation by Das Sarma and Wang [7] for wires with a two-component plasma, although the interpretation differs somewhat from that discussed here. While the tendency toward cancellation of BGR and excitonic screening is concluded in both studies, the most striking difference between the results of Ref. [7] and those here concern the unbinding of the exciton which, in an electron-hole plasma, is called the Mott transition. The authors of Ref. [7] estimate a merging of the exciton energy with the electron-hole band edge at around $n \sim 3 \times 10^{5} \mathrm{~cm}^{-1}$, where $n$ is here of course the density of electrons and holes. Even in the simple one-electron static screening approximation they find a vanishing of the exciton binding energy with density. By contrast, we find that up to the highest density considered $\left(3 \times 10^{6} \mathrm{~cm}^{-1}\right)$ the exciton remains bound. Assuming that both conclusions are correct, the implication is that it is the interaction between excitons which leads to their unbinding or, equivalently, their merging with the continuum.

In any event, the continued existence, despite screening, of a bound state of the electron with the hole in one dimension is not surprising in that in 1D any small attractive potential should bind an electron. One could expect that, at some density the screening of electrons near the Fermi surface would effectively cancel the total potential produced by the hole, thereby releasing the bound electron. However for this to occur the Fermi wavelength would need to be much smaller than the exciton radius, which is $\sim 1 a_{B}^{*}$. Our calculations have not, as yet, shown a density where such a transition of the screening cloud from states at the bottom of the Fermi sea to states at the top of the Fermi sea occurs, but should such a transition exist it would be of considerable interest theoretically.

\section{CONCLUSION}

In conclusion, we have presented results of density functional calculations for the electronic structure of modulation doped and gated T-shaped quantum wires for the case where the wire can be assumed to be translationally invariant. We have shown that the phenomenon of bandgap renormalization can be qualitatively and quantitatively understood within DFT. We have further employed a simplified model of a cylindrical quantum wire 
to examine the strength of the bound exciton state in the presence of a one dimensional electron gas. We have normalized out the band structure problem here by defining the exciton binding energy as the difference between the total energy of the wire with a localized hole and that with the hole charge (and consequently all electrons) spread uniformly along the wire. We find that the variation of the exciton binding energy with density tends to cancel the bandgap renormalization, in agreement with recent experiments. Finally, we have noted that, in this one component plasma case, to the highest densities which we have considered, no analogue of the Mott transition, i.e. no unbinding of the electron and hole occurs. Rather, the orthogonality of the free electron states with those of the bound electron(s) leads to an anti-screening behavior such that as $n_{e}$ increases a second (trion) and even a third (quadron) bound state forms at the hole.

In the future we hope to investigate the effect of dimensionality on the exciton physics by extending the calculation to include multiple 1D subbands.

\section{ACKNOWLEDGEMENTS}

I wish to thank Werner Wegscheider, Stefan Sedlmaier, Sankar Das Sarma and Elisa Molinari for helpful conversations.

[1] S. Glutsch, F. Bechstedt, W. Wegscheider and G. Schedelbeck, Phys. Rev. B 56, 4108 (1997).

[2] F. Rossi and E. Molinari, Phys. Rev. Lett. 76, 3642 (1996).

[3] D. Brinkmann and G. Fishman, Phys. Rev. B 56, 15211 (1997).

[4] W. Wegscheider, L. N. Pfeiffer, M. M. Dignam, A. Pinczuk, K. W. West, S. L. McCall and R. Hull, Phys. Rev. Lett. 71, 4071 (1993).

[5] R. Ambigapathy et al., Phys. Rev. Lett. 78, 3579 (1997).

[6] R. Zimmermann, K. Kilimann, W. D. Kraeft, D. Kremp and G. Röpke, Phys. Stat. Sol. 90, 175 (1978).
[7] S. Das Sarma and D. W. Wang, cond-mat/9905038.

[8] E. H. Hwang and S. Das Sarma, Phys. Rev. B 58, R1738 (1998).

[9] See, for example, W. L. Bloss, Jour. Appl. Phys. 65, 4789 (1989).

[10] C. Guillemot, Phys. Rev. B 31, 1428 (1985).

[11] See, for example: Electron Correlations in Molecules and Solids, Peter Fulde (Springer-Verlag, Berlin, 1995), and references therein.

[12] The detailed growth pattern of the device which we model and photoluminescence experiments in the presence of a gate-induced 1DEG will be described in a companion publication: S. Sedlmaier, M. Stopa, G. Schedelbeck, W. Wegscheider and G. Abstreiter, unpublished.

[13] For a discussion of our numerical techniques see M. Stopa, Phys. Rev. B, 54, 13767 (1996).

[14] S. Das Sarma, R. Jalabert and S. R. Eric Yang, Phys. Rev. B 39, 5516 (1989); 41, 8288 (1990).

[15] S. Schmitt-Rink, D. Chemla and D. A. B. Miller, Adv. in Phys. 38, 89 (1989); R. Cingolani and K. Ploog, ibid. 40, 535 (1991).

[16] D. M. Ceperley and B. J. Alder, Phys. Rev. Lett. 45, 566 (1980).

[17] We employ the standard treatment of the band gap, taking in particular $E_{g}=1512+1455 \eta(\mathrm{meV})$ and $V_{C}=Q_{e} E_{g}$, where the bandgap offset parameter is taken as $Q_{e}=0.6$. See Zachaw et al., Phys. Rev. B 33, 8564 (1986).

[18] M.Stopa Phys. Rev. B 53, 9595 (1996).

[19] U. Ekenberg and M. Alterelli, Phys. Rev. B 35, 7585 (1987).

[20] G. Goldoni, F. Rossi, E. Molinari and A. Fasolino, Phys. Rev. B, 55, 7110 (1997).

[21] A. Paassen, A. Zrenner, A. Efros, M. Stopa, J. Frankenberger, M. Bichler and W. Wegscheider, unpublished.

[22] G. Mahan, Many-Particle Physics (Plenum, New York, 1986).

[23] B. Vinter, Phys. Rev. B, 26, 6808 (1982).

[24] M. Stopa, Y. Aoyagi and T. Sugano, Phys. Rev. B 51, 5494 (1995).

[25] G. D. Sanders and Y. -C. Chang, Phys. Rev. B 45, 9202 (1992).

[26] R. Schilling and D. C. Mattis, Phys. Rev. Lett. 49, 808 (1982). 